\title{
Liquid-phase synthesis of 4-vinyl-1H-1,2,3-triazoles based on polyethylene glycol supported but-3-ynyl sulfonate
}

\author{
Zhe Wang, ${ }^{a}$ Li-Li Chen, ${ }^{* a}$ Xue-Chun Mao, ${ }^{b}$ Shou-Ri Sheng, ${ }^{\text {a,b }}$ and Xiao-Ling Liu *a,b \\ ${ }^{a}$ National Research Center for Carbohydrate Synthesis, Jiangxi Normal University \\ Nanchang 330022, P. R. China \\ ${ }^{b}$ Key Laboratory of Functional Small Organic Molecule, Ministry of Education \\ Jiangxi Normal University, Nanchang 330022, P. R. China \\ E-mail: chenlilijxsd@163.com, liuxiaoling@jxnu.edu.cn
}

DOI: http://dx.doi.org/10.3998/ark.5550190.p009.491

\begin{abstract}
An efficient liquid-phase synthesis of 4-vinyl-1H-1,2,3-triazole derivatives is developed from soluble polyethylene glycol (PEG) supported but-3-yn-1-yl sulfonate. This procedure affords the target compounds in moderate-to-excellent yields (58-91\%) with mild reaction conditions and a facile work-up procedure.
\end{abstract}

Keywords: Liquid-phase synthesis, 4-vinyl-1H-1,2,3-triazoles, PEG-supported but-3-yn-1-yl sulfonate, click chemistry; elimination reaction

\section{Introduction}

1,2,3-Triazoles, as an important class of nitrogen-containing heterocycles, have gained great attention and have been applied widely in pharmaceuticals, agrochemicals, dyes, corrosion inhibitors, biochemicals, polymers, and functional materials. ${ }^{1-3}$ Since the Sharpless ${ }^{4}$ and Meldel ${ }^{5}$ groups independently discovered the copper-catalyzed Huisgen 1,3-dipolar cycloaddition (CuAAC) reaction between azides and terminal alkynes, 'click chemistry' has received growing interest in the regioselective synthesis of 1,4-disubstituted 1,2,3-triazoles. Following this pioneering work, many synthetic methods for producing this ring system have been developed for various purposes. ${ }^{6-13}$ Among them, vinyl-substituted 1,2,3-triazoles are useful vinyl monomers, which have been demonstrated to take advantage of the 1,2,3-triazole subunit and combine the features found in classical monomers, such as aromaticity, polarity, and structural diversity inherent in styrenics, vinylpyridines, and acrylates, respectively, into a single building block. ${ }^{14}$ There are, however, only a few reports concerning the synthesis of vinyl-substituted 1,2,3triazoles. ${ }^{15-19}$ Even up to now, only a few methods for preparation of some typical vinyl-substituted 
1,2,3-triazoles, such as 1-vinyl-5,6-dihydro[1,2,3]triazolo[5,1-a]isoquinoline, ${ }^{20}$ 1-benzyl-4-vinyl$1 H$-1,2,3-triazole ${ }^{21}$ and 5-vinyl-1H-1,2,3-triazoles ${ }^{22}$ have been described. Therefore, the development of a simple and practical procedure to access vinyl-substituted 1,2,3-triazoles is still highly desirable.

Recently, there has been a considerable growth in interest in the use of soluble polymersupported catalysts and reagents in organic synthesis because of their low cost, ease of preparation and simple work-up ${ }^{23,24}$ Liquid-phase organic synthesis (LPOS) on soluble polymers combines the advantages of classical homogeneous solution methodology (high reactivity and simple analytical procedures) with those of solid-phase organic synthesis (SPOS) (use of excess reagents, easy isolation and purification of the final products and high stability of the system polymersupported molecule). Herein, in continuation of our studies on the use polyethylene glycol (PEG) as support in LPOS, ${ }^{25}$ we report a novel method for the soluble polymer-supported synthesis of 4-vinyl-1H-1,2,3-triazoles from PEG-supported but-3-yn-1-yl sulfonate and azides, involving sequential click and elimination reactions, as depicted in Scheme 1.

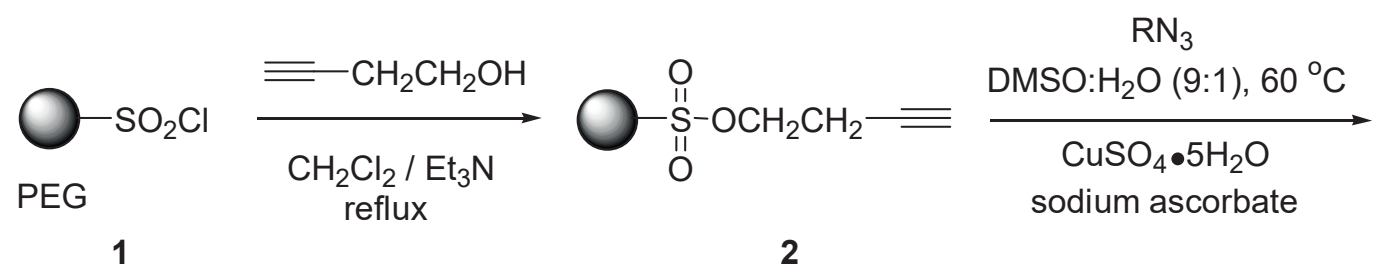

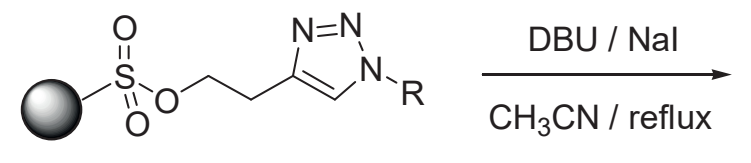

3<smiles>[R]n1cc(C=C)nn1</smiles>

4

Scheme 1. PEG supported synthetic route to 4-vinyl-1H-1,2,3-triazoles.

\section{Results and Discussion}

As shown in Scheme 1, treatment of the difunctionalised PEG-sulfonyl chloride (1) with but-3yn-1-ol in dichloromethane in the presence of triethylamine gave rise to the corresponding PEGsupported but-3-yn-1-yl sulfonate (2), where the polymer species were easily obtained through precipitation by addition of propan-2-ol to the reaction mixture and then simple filtration. The linking reaction to the PEG support was monitored by the infrared absorption bands at $1371 \mathrm{~cm}^{-1}$ (S-O stretch of $\left.\mathrm{SO}_{2} \mathrm{C} 1\right)$, which was shifted to $1353 \mathrm{~cm}^{-1}\left(-\mathrm{SO}_{2}-\mathrm{O}-\right)$, as well as by the appearance of a typical carbon-carbon triple bond absorption at $2122 \mathrm{~cm}^{-1}$. Then, the [3+2] cycloaddition reaction conditions of $\mathbf{2}$ with benzyl azide as a model substrate was investigated. Numerous experimental conditions have been reported to promote the $\mathrm{CuAAC}$ reaction. ${ }^{3,6-8} \mathrm{On}$ the basis of those results, the $\mathrm{Cu}(\mathrm{I})$ catalyst generated in situ by reduction of $\mathrm{CuSO}_{4} \cdot 5 \mathrm{H}_{2} \mathrm{O}$ was chosen in our 
experiment since this method was less costly and was reported to give purer material than some $\mathrm{Cu}(\mathrm{I})$ salts that are commercially available. Next, various polar solvents such as $t$ - $\mathrm{BuOH}, \mathrm{DMF}$ and DMSO, and their different combinations, as well as the amount of catalyst, reaction temperature and reaction time, were optimized. After varying the reaction conditions, the 1,3dipolar cycloaddition of 2 with benzyl azide proceeded smoothly in DMSO/ $\mathrm{H}_{2} \mathrm{O}$ (9:1) in the presence of catalyst $(20 \mathrm{mmol} \%)$ at $60{ }^{\circ} \mathrm{C}$ for 10 hours to afford the PEG-supported 2-(4triazolyl)ethyl sulfonate (3a). This could also be monitored conveniently by IR spectrometry and precisely, whereby the characteristic absorption of the carbon-carbon triple bond $\left(2122 \mathrm{~cm}^{-1}\right)$ had been distinctly shrunk after $6 \mathrm{~h}$ of reaction time, and disappeared completely after a further foyr hours of reaction time.

Table 1. Yields of 4-vinyl-1H-1,2,3-triazoles (4a-4k)

\begin{tabular}{llll}
\hline Entry & $\mathrm{R}$ & Product & Yield (\%) \\
\hline 1 & $\mathrm{C}_{6} \mathrm{H}_{5} \mathrm{CH}_{2}$ & $\mathbf{4 a}$ & 88 \\
2 & $4-\mathrm{MeOC}_{6} \mathrm{H}_{4} \mathrm{CH}_{2}$ & $\mathbf{4 b}$ & 86 \\
3 & $4-\mathrm{BrC}_{6} \mathrm{H}_{4} \mathrm{CH}_{2}$ & $\mathbf{4 c}$ & 83 \\
4 & $\mathrm{CH}_{3}\left(\mathrm{CH}_{2}\right)_{2} \mathrm{CH}_{2}$ & $\mathbf{4 d}$ & 84 \\
5 & $\mathrm{C}_{6} \mathrm{H}_{5}$ & $\mathbf{4 e}$ & 88 \\
6 & $4-\mathrm{MeOC}_{6} \mathrm{H}_{4}$ & $\mathbf{4 f}$ & 90 \\
7 & $3-\mathrm{MeC}_{6} \mathrm{H}_{4}$ & $\mathbf{4 g}$ & 91 \\
8 & $2-\mathrm{MeC}_{6} \mathrm{H}_{4}$ & $\mathbf{4 h}$ & 62 \\
9 & $2-\mathrm{ClC}_{6} \mathrm{H}_{4}$ & $\mathbf{4 i}$ & 58 \\
10 & $4-\mathrm{BrC}_{6} \mathrm{H}_{4}$ & $\mathbf{4 j}$ & 86 \\
11 & $4-\mathrm{O}_{2} \mathrm{NC}_{6} \mathrm{H}_{4}$ & $\mathbf{4 k}$ & 85 \\
\hline
\end{tabular}

${ }^{a}$ All yields refer to the isolated products after purification based on $\mathbf{1}$.

Upon completion of the optimal click reaction conditions, PEG immobilized intermediate 3a was separated by simple filtration after addition of diethyl ether to the reaction mixtures. Finally, conditions for cleavage from the PEG support were tested by employing different bases such as 1,8-diazabicyclo[5.4.0] undec-7-ene (DBU) and potassium $t$-butoxide in various polar and nonpolar solvents. It was found that the desired product, 1-benzyl-4-vinyl-1 $H$-1,2,3-triazole (4a) was obtained in $88 \%$ isolated yield by treatment of $\mathbf{3 a}$ with $\mathrm{NaI} / \mathrm{DBU}$ in refluxing acetonitrile for 45 min. The structure of $4 \mathbf{a}$ was confirmed on the basis of its ${ }^{1} \mathrm{H}$ and ${ }^{13} \mathrm{C}$ NMR spectra. Thus, compound 4a displayed a characteristic singlet at $\delta=7.45 \mathrm{ppm}$ for the triazolyl C-5 $-\mathrm{H}$ proton, and a singlet signal for the $N-\mathrm{CH}_{2}$ protons appearing at $\delta=5.49 \mathrm{ppm}$. Signals for the vinyl protons as a double doublet were observed at $\delta=6.65,5.85$ and $5.30 \mathrm{ppm}$, respectively. Additionally, the melting point of $4 \mathbf{a}$ was in agreement with the reported value. ${ }^{21}$

To demonstrate further the convenience of this protocol, the substrate scope of various azides was investigated. As exemplified in Table 1, reaction with both alkyl azides (Table 1, entries 1- 
4) and aryl azides (Table 1, entries 5-11) proceeded smoothly and the corresponding products $\mathbf{4 a -}$ $\mathbf{4} \mathbf{k}$ were obtained in moderate to excellent yields. Among them, benzylic azides (Table 1, entries 1-3) and primary azides (Table 1, entry 4) were found to react readily to form the desired triazoles in high yields (83-88\%) and aryl azides bearing either electron-donating (Table 1, entries 6-8) or electron-withdrawing (Table 1, entries 9-11) substituents could also be used efficiently with 58$91 \%$ yields. However, it must be pointed out that aryl azides with an electron-donating or electronwithdrawing substituent at an ortho-position (Table 1, entries 8,9), gave the corresponding triazoles (4h and $\mathbf{4 i})$ in relatively low yields respectively, even when the click reaction time was extended to 24 hours, possibly due to the stereo-hindrance effect of the substituents.

In the ${ }^{1} \mathrm{HNMR}$ spectra of the products $\mathbf{4 a}-\mathbf{4 k}$, the resonances of the vinyl protons were seen as a double doublet at $\delta=6.56-6.81\left(-\mathrm{CH}=\mathrm{CH}_{2}\right), 5.82-6.02\left(=\mathrm{CH}_{\text {cis }} H_{\text {trans }}\right)$ and $5.18-5.41$ $\left(=\mathrm{C} H_{\text {cis }} \mathrm{H}_{\text {trans }}\right)$, respectively. All products had a one-proton singlet at about $\delta=7.42-7.94 \mathrm{ppm}$ assignable to the triazolyl $\mathrm{C}-5-\mathrm{H}$ proton. These signals clearly demonstrate the formation of the target molecules. Further support was obtained from their ${ }^{13} \mathrm{C}$ NMR spectra.

\section{Conclusions}

In summary, a novel and efficient procedure for the liquid-phase synthesis of 1-substituted-4vinyl-1H-1,2,3-triazoles has been developed using PEG-supported but-3-yn-1-yl sulfonate reagent, which involved sequential click and elimination reactions. This method gives these interesting compounds in moderate to excellent yields with simple work-up and mild reaction conditions.

\section{Experimental Section}

General. Melting points are uncorrected. NMR spectra were recorded on a Bruker Avance (400 $\mathrm{MHz}$ ) spectrometer, using $\mathrm{CDCl}_{3}$ as the solvent and tetramethylsilane (TMS) as internal standard. FTIR spectra were taken on a Perkin-Elmer SP One FTIR spectrophotometer. Microanalyses were performed with a Carlo Erba 1106 Elemental Analyzer. Difunctionalised polyethylene glycol (PEG) supported sulfonyl chloride (1) (loading: $0.46 \mathrm{mmol} \mathrm{Cl} / \mathrm{g}$ ) was prepared from PEG polymer (average mass 4000) according to our reported method. ${ }^{26}$ The azides were prepared from reaction of sodium azide with the corresponding alkyl halides or aryl halides according to the procedures given in the literature. ${ }^{27}$ The other reagents were purchased from commercial suppliers and used without further purification. Column chromatography was performed on silica gel (60-120 mesh) using ethyl acetate/hexane mixtures as the eluent.

Preparation of PEG-supported but-3-ynyl sulfonate (2). To a solution of 3-butyn-1-ol (5.0 $\mathrm{mmol})$ in anhydrous dichloromethane $(10.0 \mathrm{~mL})$ was added PEG 4000 disulfonyl chloride (1) (2.2 $\mathrm{g}, 0.05 \mathrm{mmol})$ and $\mathrm{Et}_{3} \mathrm{~N}(2.5 \mathrm{mmol})$, and the reaction mixture was heated at reflux for $8 \mathrm{~h}$ under an $\mathrm{N}_{2}$ atmosphere. The solvent was removed in vacuo, and the crude product dissolved in hot 
propan-2-ol $(100 \mathrm{~mL})$. The precipitation step was repeated, the combined precipitates washed with propan-2-ol $(10 \mathrm{~mL})$, and $\mathrm{Et}_{2} \mathrm{O}(2 \times 10 \mathrm{~mL})$, and then dried in vacuo to afford PEG-supported but3-yn-1-yl sulfonate (2) as a white solid in nearly quantitative yield. IR ( $\mathrm{KBr}$ ): $v_{\max }=3321,2945,2886,2122,1455,1353,1245,1170,695 \mathrm{~cm}^{-1}$.

General procedure for the preparation of 4 -vinyl-1H-1,2,3-triazoles (4a-4k). To a stirred solution of 2 prepared as above, azides $(3.00 \mathrm{mmol})$ and sodium ascorbate $(38 \mathrm{mg}, 20 \mathrm{mmol} \%)$ in $\mathrm{DMSO} / \mathrm{H}_{2} \mathrm{O}(9: 1,10 \mathrm{~mL})$ was added $\mathrm{CuSO}_{4} \cdot 5 \mathrm{H}_{2} \mathrm{O}(50 \mathrm{mg}, 20 \mathrm{mmol} \%)$, and the mixture was heated at $60{ }^{\circ} \mathrm{C}$ for $10 \mathrm{~h}$. After completion of the reaction, the reaction mixture was cooled and the diethyl ether $(100 \mathrm{~mL})$ was added to cause precipitation of PEG-supported intermediate 3. To complete the precipitation, the suspension was left at $0{ }^{\circ} \mathrm{C}$ for another $30 \mathrm{~min}$. The white precipitate was collected, washed with diethyl ether $(2 \times 10 \mathrm{~mL})$ and dried in vacuo. Then, to a solution of $\mathbf{3}$ in $\mathrm{MeCN}$ (10 mL), NaI (450 mg, $3.00 \mathrm{mmol})$ and DBU (300 mg, $2.00 \mathrm{mmol}$ ) were added, and the reaction mixture was heated at reflux for 40-50 min under an $\mathrm{N}_{2}$ atmosphere. After this, the solvent was removed under vacuum, and diethyl ether $(100 \mathrm{~mL})$ was added with vigorous stirring and the mixture was cooled to $0{ }^{\circ} \mathrm{C}$. The recovered PEG-bound sulfonic acid salt was collected by filtration, washed with cold diethyl ether $(2 \times 10 \mathrm{~mL})$. The filtrate was washed water (each of 10 $\mathrm{mL}$ ), dried over anhydrous magnesium sulfate. After evaporation of the solvent, the residue was subjected to column chromatography (silica gel; hexane/EtOAc, 10:1) to afforded pure target compounds $\mathbf{4 a}-\mathbf{4} \mathbf{k}$.

1-Benzyl-4-vinyl-1H-1,2,3-triazole (4a). White solid, mp 37-38 ${ }^{\circ} \mathrm{C}$ (lit. $\left.{ }^{16,17,21} 36-39^{\circ} \mathrm{C}\right) ;{ }^{1} \mathrm{H}$ NMR $\left(\mathrm{CDCl}_{3}, 400 \mathrm{MHz}\right): \delta_{\mathrm{H}}=7.45(\mathrm{~s}, 1 \mathrm{H}$, triazolyl $H), 7.44-7.38(\mathrm{~m}, 3 \mathrm{H}, \mathrm{Ph} H), 7.30-7.27(\mathrm{~m}, 2 \mathrm{H}$, $\mathrm{Ph} H$ ), 6.65 (dd, $\left.J 17.8,10.8 \mathrm{~Hz}, 1 \mathrm{H}, \mathrm{CH}=\mathrm{CH}_{2}\right), 5.85$ (d, $J 17.8 \mathrm{~Hz}, 1 \mathrm{H}$, trans $\left.\mathrm{CH}=\mathrm{CH}_{2}\right), 5.49$ (s, $\left.2 \mathrm{H}, \mathrm{CH}_{2} \mathrm{~N}\right), 5.30\left(\mathrm{~d}, J 10.8 \mathrm{~Hz}, 1 \mathrm{H}\right.$, cis $\left.\mathrm{CH}=\mathrm{CH}_{2}\right) ;{ }^{13} \mathrm{C} \mathrm{NMR}\left(\mathrm{CDCl}_{3}, 100 \mathrm{MHz}\right): \delta_{\mathrm{C}}=146.8$ $(\mathrm{NCH}=C), 134.8\left(\mathrm{Ph}, \mathrm{CCH}_{2} \mathrm{~N}\right), 129.3(o-\mathrm{Ph}, 2 \mathrm{C}), 128.8\left(\mathrm{CH}=\mathrm{CH}_{2}\right), 128.2(m-\mathrm{Ph}, 2 \mathrm{C}), 125.8(p-$ $\mathrm{Ph}), 120.3\left(\mathrm{CH}=\mathrm{CH}_{2}\right), 116.1(\mathrm{NCH}=\mathrm{C}), 54.2\left(\mathrm{CH}_{2} \mathrm{~N}\right)$.

1-(4-Methoxybenzyl)-4-vinyl-1H-1,2,3-triazole (4b). White solid, mp $46-47^{\circ} \mathrm{C}$ (lit. ${ }^{16,17} 46-47$ $\left.{ }^{\circ} \mathrm{C}\right) ;{ }^{1} \mathrm{H} \mathrm{NMR}\left(\mathrm{CDCl}_{3}, 400 \mathrm{MHz}\right): \delta_{\mathrm{H}}=7.42(\mathrm{~s}, 1 \mathrm{H}$, triazolyl $H), 7.25(\mathrm{~d}, J 8.0 \mathrm{~Hz}, 2 \mathrm{H}, o-\mathrm{Ph} H)$, $6.95(\mathrm{~d}, J 8.0 \mathrm{~Hz}, 2 \mathrm{H}, m-\mathrm{Ph} H), 6.66\left(\mathrm{dd}, J 17.8,10.6 \mathrm{~Hz}, 1 \mathrm{H}, \mathrm{CH}=\mathrm{CH}_{2}\right), 5.84(\mathrm{~d}, J 17.8 \mathrm{~Hz}, 1 \mathrm{H}$, trans $\left.\mathrm{CH}=\mathrm{CH}_{2}\right), 5.47\left(\mathrm{~s}, 2 \mathrm{H}, \mathrm{CH}_{2} \mathrm{~N}\right), 5.30\left(\mathrm{~d}, J 10.6 \mathrm{~Hz}, 1 \mathrm{H}\right.$, cis $\left.\mathrm{CH}=\mathrm{CH}_{2}\right), 3.84\left(\mathrm{~s}, 3 \mathrm{H}, \mathrm{OCH}_{3}\right)$; ${ }^{13} \mathrm{C} \mathrm{NMR}\left(\mathrm{CDCl}_{3}, 100 \mathrm{MHz}\right): \delta_{\mathrm{C}}=160.3\left(\mathrm{Ph}, \mathrm{CH}_{3} \mathrm{OC}\right), 146.7(\mathrm{NCH}=C), 129.9(o-\mathrm{Ph}, 2 \mathrm{C}), 126.7$ $\left(\mathrm{Ph}, \mathrm{CCH}_{2} \mathrm{~N}\right), 128.7\left(\mathrm{CH}=\mathrm{CH}_{2}\right), 120.3(\mathrm{NCH}=\mathrm{C}), 116.1\left(\mathrm{CH}=\mathrm{CH}_{2}\right), 114.8(m-\mathrm{Ar}, 2 \mathrm{C}), 55.8$ $\left(\mathrm{CH}_{3} \mathrm{O}\right), 53.8\left(\mathrm{CH}_{2} \mathrm{~N}\right)$.

1-(4-Bromobenzyl)-4-vinyl-1H-1,2,3-triazole (4c). White solid, mp $134-135{ }^{\circ} \mathrm{C}$ (lit. ${ }^{17} 135-137$ $\left.{ }^{\circ} \mathrm{C}\right) ;{ }^{1} \mathrm{H}$ NMR $\left(\mathrm{CDCl}_{3}, 400 \mathrm{MHz}\right): \delta_{\mathrm{H}}=7.52(\mathrm{~s}, 1 \mathrm{H}$, triazolyl $H), 7.70(\mathrm{~d}, J 6.8 \mathrm{~Hz}, 2 \mathrm{H}, m-\mathrm{Ph} H)$, $7.45(\mathrm{~d}, J 6.8 \mathrm{~Hz}, 2 \mathrm{H}, o-\mathrm{Ph} H), 6.68\left(\mathrm{dd}, J 18.2,10.2 \mathrm{~Hz}, 1 \mathrm{H}, \mathrm{CH}=\mathrm{CH}_{2}\right), 5.82(\mathrm{~d}, J 18.2 \mathrm{~Hz}, 1 \mathrm{H}$, trans $\left.\mathrm{CH}=\mathrm{CH}_{2}\right), 5.23\left(\mathrm{~s}, 2 \mathrm{H}, \mathrm{CH}_{2} \mathrm{~N}\right), 5.35\left(\mathrm{~d}, J 10.2 \mathrm{~Hz}, 1 \mathrm{H}\right.$, cis $\left.\mathrm{CH}=\mathrm{CH}_{2}\right) ;{ }^{13} \mathrm{C} \mathrm{NMR}\left(\mathrm{CDCl}_{3}, 100\right.$ 
MHz): $\delta_{\mathrm{C}}=147.0(\mathrm{NCH}=C), 134.6(m-\mathrm{Ph}, 2 \mathrm{C}), 132.4(o-\mathrm{Ph}, 2 \mathrm{C}), 129.8\left(\mathrm{Ph}, C_{C H} \mathrm{~N}\right), 129.0$ $\left(\mathrm{CH}=\mathrm{CH}_{2}\right), 123.1(\mathrm{Ph}, \mathrm{BrC}), 120.5(\mathrm{NCH}=\mathrm{C}), 116.3\left(\mathrm{CH}=\mathrm{CH}_{2}\right), 53.5\left(\mathrm{CH}_{2} \mathrm{~N}\right)$.

1-(n-Butyl)-4-vinyl-1 H-1,2,3-triazole (4d). Colorless oil (lit. $\left.{ }^{17}\right) ;{ }^{1} \mathrm{H} \mathrm{NMR}\left(\mathrm{CDCl}_{3}, 400 \mathrm{MHz}\right): \delta_{\mathrm{H}}$ $=7.48(\mathrm{~s}, 1 \mathrm{H}$, triazolyl $H), 6.56\left(\mathrm{dd}, J 17.8,10.0 \mathrm{~Hz}, 1 \mathrm{H}, \mathrm{CH}=\mathrm{CH}_{2}\right), 5.85(\mathrm{~d}, J 17.8 \mathrm{~Hz}, 1 \mathrm{H}$, trans $\left.\mathrm{CH}=\mathrm{CH}_{2}\right), 5.18\left(\mathrm{~d}, J 10.0 \mathrm{~Hz}, 1 \mathrm{H}\right.$, cis $\left.\mathrm{CH}=\mathrm{CH}_{2}\right), 4.20\left(\mathrm{t}, J 7.2 \mathrm{~Hz}, 2 \mathrm{H}, \mathrm{CH}_{2} \mathrm{~N}\right), 1.75-1.71(\mathrm{~m}, 2 \mathrm{H}$, $\left.\mathrm{CH}_{2} \mathrm{CH}_{2} \mathrm{~N}\right), 1.25-1.20\left(\mathrm{~m}, 2 \mathrm{H}, \mathrm{CH}_{2} \mathrm{CH}_{3}\right), 0.82$ (t, $\left.J 7.2 \mathrm{~Hz}, 3 \mathrm{H}, \mathrm{CH}_{3}\right) ;{ }^{13} \mathrm{C} \mathrm{NMR}\left(\mathrm{CDCl}_{3}, 100\right.$ $\mathrm{MHz}): \delta_{\mathrm{C}}=146.2(\mathrm{NCH}=C), 125.6\left(\mathrm{CH}=\mathrm{CH}_{2}\right), 120.6(\mathrm{NCH}=\mathrm{C}), 115.8\left(\mathrm{CH}=\mathrm{CH}_{2}\right), 49.7\left(\mathrm{CH}_{2} \mathrm{~N}\right)$, $32.3\left(\mathrm{CH}_{2} \mathrm{CH}_{2} \mathrm{~N}\right), 19.8\left(\mathrm{CH}_{2} \mathrm{CH}_{3}\right), 13.3\left(\mathrm{CH}_{3}\right)$.

1-Phenyl-4-vinyl-1H-1,2,3-triazole (4e). White solid, mp 41-42 ${ }^{\circ} \mathrm{C}$ (lit. ${ }^{17} 40-42{ }^{\circ} \mathrm{C}$ ); ${ }^{1} \mathrm{H}$ NMR $\left(\mathrm{CDCl}_{3}, 400 \mathrm{MHz}\right): \delta_{\mathrm{H}}=7.94(\mathrm{~s}, 1 \mathrm{H}$, triazolyl $H), 7.73(\mathrm{~d}, J 7.4 \mathrm{~Hz}, 2 \mathrm{H}, o-\mathrm{Ph} H), 7.55(\mathrm{t}, J 15.5$ $\mathrm{Hz}, 2 \mathrm{H}, m-\mathrm{Ph} H), 7.44(\mathrm{t}, J 8.1 \mathrm{~Hz}, 1 \mathrm{H}, p-\mathrm{Ph} H), 6.80$ (dd, $\left.J 18.0,10.2 \mathrm{~Hz}, 1 \mathrm{H}, \mathrm{CH}=\mathrm{CH}_{2}\right), 6.00$ (d, $J 18.0 \mathrm{~Hz}, 1 \mathrm{H}$, trans $\left.\mathrm{CH}=\mathrm{CH}_{2}\right), 5.42\left(\mathrm{~d}, J 10.2 \mathrm{~Hz}, 1 \mathrm{H}\right.$, cis $\left.\mathrm{CH}=\mathrm{CH}_{2}\right) ;{ }^{13} \mathrm{C} \mathrm{NMR}\left(\mathrm{CDCl}_{3}, 100\right.$ $\mathrm{MHz}): \delta_{\mathrm{C}}=148.7(\mathrm{NCH}=C), 129.8(\mathrm{NCH}=\mathrm{C}), 128.7(\mathrm{Ph}, \mathrm{CN}), 125.8\left(\mathrm{CH}=\mathrm{CH}_{2}\right), 125.3(o-\mathrm{Ph}$, 2C), 120.5 ( $m-\mathrm{Ph}, 2 \mathrm{C}), 118.5$ ( $p-\mathrm{Ph}, \mathrm{C}), 116.7\left(\mathrm{CH}=\mathrm{CH}_{2}\right)$.

1-(4-Methoxyphenyl)-4-vinyl-1H-1,2,3-triazole (4f). White solid, mp $66-67{ }^{\circ} \mathrm{C} ;{ }^{1} \mathrm{H}$ NMR $\left(\mathrm{CDCl}_{3}, 400 \mathrm{MHz}\right): \delta_{\mathrm{H}}=7.65(\mathrm{~s}, 1 \mathrm{H}$, triazolyl $H), 7.55(\mathrm{~d}, J 8.4 \mathrm{~Hz}, 2 \mathrm{H}, o-\mathrm{Ph} H), 6.94(\mathrm{~d}, J 8.4 \mathrm{~Hz}$, $2 \mathrm{H}, m-\mathrm{Ph} H), 6.71\left(\mathrm{dd}, J 18.4,10.4 \mathrm{~Hz}, 1 \mathrm{H}, \mathrm{CH}=\mathrm{CH}_{2}\right), 5.94\left(\mathrm{~d}, J 18.4 \mathrm{~Hz}, 1 \mathrm{H}\right.$, trans $\left.\mathrm{CH}=\mathrm{CH}_{2}\right)$, $5.43\left(\mathrm{~d}, J 10.4 \mathrm{~Hz}, 1 \mathrm{H}\right.$, cis $\left.\mathrm{CH}=\mathrm{CH} H_{2}\right), 3.79\left(\mathrm{~s}, 3 \mathrm{H}, \mathrm{OCH}_{3}\right) ;{ }^{13} \mathrm{C} \mathrm{NMR}\left(\mathrm{CDCl}_{3}, 100 \mathrm{MHz}\right): \delta_{\mathrm{C}}=159.7$ $\left(\mathrm{Ph}, \mathrm{CH}_{3} \mathrm{OC}\right), 148.5(\mathrm{NCH}=C), 130.2(\mathrm{NCH}=\mathrm{C}), 129.7(\mathrm{Ph}, \mathrm{CN}), 125.7\left(\mathrm{CH}=\mathrm{CH}_{2}\right), 121.7(o-\mathrm{Ph}$, 2C), $116.7\left(\mathrm{CH}=\mathrm{CH}_{2}\right), 114.3(m-\mathrm{Ph}, 2 \mathrm{C}), 55.6\left(\mathrm{CH}_{3} \mathrm{O}\right)$; IR $(\mathrm{KBr}): v_{\max }=3071,2926,2864,1641$, 1545, 1496, 1458, 1378, 1221, 996, 910, 826, $720 \mathrm{~cm}^{-1}$; Anal. Calcd for $\mathrm{C}_{11} \mathrm{H}_{11} \mathrm{~N}_{3} \mathrm{O}: \mathrm{C}$ 65.66, $\mathrm{H}$ 5.51, N 20.88; found: C 65.45 H, 5.67, N 20.75.

1-(3-Methylphenyl)-4-vinyl-1H-1,2,3-triazole (4g). Yellow oil; ${ }^{1} \mathrm{H} \mathrm{NMR}\left(\mathrm{CDCl}_{3}, 400 \mathrm{MHz}\right): \delta_{\mathrm{H}}$ $=7.77(\mathrm{~s}, 1 \mathrm{H}$, triazolyl $H), 7.75(\mathrm{~s}, 1 \mathrm{H}, \mathrm{Ph}-2 H), 7.45(\mathrm{~d}, J 7.6 \mathrm{~Hz}, 1 \mathrm{H}, \mathrm{Ph}-6 H), 7.34(\mathrm{~d}, J 8.0 \mathrm{~Hz}$, 1H, Ph-4H), 7.21 (d, J 7.2 Hz, 1H, Ph-5H), 6.73 (dd, J 18.4, $\left.10.8 \mathrm{~Hz}, 1 \mathrm{H}, \mathrm{CH}=\mathrm{CH}_{2}\right), 5.93$ (d, $J$ $18.4 \mathrm{~Hz}, 1 \mathrm{H}$, trans $\left.\mathrm{CH}=\mathrm{CH}_{2}\right), 5.44\left(\mathrm{~d}, J 10.8 \mathrm{~Hz}, 1 \mathrm{H}\right.$, cis $\left.\mathrm{CH}=\mathrm{CH}_{2}\right), 2.40\left(\mathrm{~s}, 3 \mathrm{H}, \mathrm{CH}_{3}\right) ;{ }^{13} \mathrm{C} \mathrm{NMR}$ $\left(\mathrm{CDCl}_{3}, 100 \mathrm{MHz}\right): \delta_{\mathrm{C}}=148.8(\mathrm{NCH}=C), 139.8(\mathrm{Ph}-3 C), 136.8(\mathrm{Ph}-2 C), 130.6(\mathrm{NCH}=\mathrm{C}), 129.5$ (Ph-1C), $128.9(\mathrm{Ph}-6 C), 126.0\left(\mathrm{CH}=\mathrm{CH}_{2}\right), 121.5(\mathrm{Ph}-4 C), 117.7(\mathrm{Ph}-5 C), 116.8\left(\mathrm{CH}=\mathrm{CH}_{2}\right), 20.9$ $\left(\mathrm{CH}_{3}\right)$; IR (film): $v_{\max }=3114,3069,2925,2872,1639,1550,1496,1437,1376,1224,996,865$, $694 \mathrm{~cm}^{-1}$; Calcd for $\mathrm{C}_{11} \mathrm{H}_{11} \mathrm{~N}_{3}$ : C 71.33, H 5.99, N 22.69; found: C 71.07, H 6.20, N 22.53.

1-(2-Methylphenyl)-4-vinyl-1H-1,2,3-triazole (4h). Yellow oil; ${ }^{1} \mathrm{H} \mathrm{NMR}\left(\mathrm{CDCl}_{3}, 400 \mathrm{MHz}\right): \delta_{\mathrm{H}}$ $=7.70(\mathrm{~s}, 1 \mathrm{H}$, triazolyl $H), 7.44-7.31(\mathrm{~m}, 3 \mathrm{H}, \mathrm{Ph} H), 7.21(\mathrm{~d}, J 8.0 \mathrm{~Hz}, 1 \mathrm{H}, \mathrm{Ph}-3 H), 6.86(\mathrm{dd}, J$ $\left.18.4,10.4 \mathrm{~Hz}, 1 \mathrm{H}, \mathrm{CH}=\mathrm{CH}_{2}\right), 5.93\left(\mathrm{~d}, J 18.4 \mathrm{~Hz}, 1 \mathrm{H}\right.$, trans $\left.\mathrm{CH}=\mathrm{CH}_{2}\right), 5.48(\mathrm{~d}, J 10.4 \mathrm{~Hz}, 1 \mathrm{H}$, cis $\left.\mathrm{CH}=\mathrm{CH}_{2}\right), 2.33\left(\mathrm{~s}, 3 \mathrm{H}, \mathrm{CH}_{3}\right) ;{ }^{13} \mathrm{C} \mathrm{NMR}\left(\mathrm{CDCl}_{3}, 100 \mathrm{MHz}\right): \delta_{\mathrm{C}}=148.4(\mathrm{NCH}=\mathrm{C}), 136.8(\mathrm{Ph}-2 \mathrm{C})$, $132.9(\mathrm{Ph}-6 C), 131.2(\mathrm{Ph}-5 C), 130.6(\mathrm{NCH}=\mathrm{C}), 129.5(\mathrm{Ph}-1 C), 125.9\left(\mathrm{CH}=\mathrm{CH}_{2}\right), 125.3(\mathrm{Ph}-4 C)$, 
$121.7(\mathrm{Ph}-3 \mathrm{C}), 116.8\left(\mathrm{CH}=\mathrm{CH}_{2}\right), 20.9\left(\mathrm{CH}_{3}\right)$; IR (film): $v_{\max }=3114,3086,2926,2874,1642$, 1550, 1496, 1455, 1377, 1224, 995, 868, $695 \mathrm{~cm}^{-1}$; Calcd for $\mathrm{C}_{11} \mathrm{H}_{11} \mathrm{~N}_{3}$ : C 71.33, H 5.99, N 22.69; found: C 71.09, H 6.21, N 22.55.

1-(2-Chlorophenyl)-4-vinyl-1H-1,2,3-triazole (4i). Yellow oil; ${ }^{1} \mathrm{H}$ NMR $\left(\mathrm{CDCl}_{3}, 400 \mathrm{MHz}\right): \delta_{\mathrm{H}}$ $=7.77(\mathrm{~s}, 1 \mathrm{H}$, triazolyl H), $7.73(\mathrm{~d}, J 7.2 \mathrm{~Hz}, 1 \mathrm{H}, \mathrm{Ph}-3 H), 7.49-7.42(\mathrm{~m}, 2 \mathrm{H}, \mathrm{Ph} H), 7.34(\mathrm{t}, J 6.8$ $\mathrm{Hz}, 1 \mathrm{H}, \mathrm{Ph}-5 H), 6.77$ (dd, $\left.J 17.8,10.4 \mathrm{~Hz}, 1 \mathrm{H}, \mathrm{CH}=\mathrm{CH}_{2}\right), 5.95\left(\mathrm{~d}, J 17.8 \mathrm{~Hz}, 1 \mathrm{H}\right.$, trans $\left.\mathrm{CH}=\mathrm{CH}_{2}\right)$, $5.49\left(\mathrm{~d}, J 10.4 \mathrm{~Hz}, 1 \mathrm{H}\right.$, cis $\left.\mathrm{CH}=\mathrm{CH}_{2}\right) ;{ }^{13} \mathrm{C} \mathrm{NMR}\left(\mathrm{CDCl}_{3}, 100 \mathrm{MHz}\right): \delta_{\mathrm{C}}=149.1(\mathrm{NCH}=C), 133.4$ (Ph-2C), 131.7 (Ph-6C), 130.9 (Ph-4C), $130.6(\mathrm{NCH}=\mathrm{C}), 129.8(\mathrm{Ph}-1 C), 128.2(\mathrm{Ph}-3 C), 127.5$ (Ph-5C), $125.9\left(\mathrm{CH}=\mathrm{CH}_{2}\right), 116.2\left(\mathrm{CH}=\mathrm{CH}_{2}\right)$; IR (film): $v_{\max }=3122,3096,2928,2875,1644$, 1550, 1495, 1441, 1225, 1001, 906, 817, $696 \mathrm{~cm}^{-1}$; Calcd for $\mathrm{C}_{10} \mathrm{H}_{8} \mathrm{~N}_{3} \mathrm{Cl}$ : C 58.41, H 3.92, N 20.43; found: C 58.21, H 4.11, N 20.30.

1-(4-Bromophenyl)-4-vinyl-1H-1,2,3-triazole (4j). Pale yellow solid, mp $141-142{ }^{\circ} \mathrm{C}\left(\right.$ lit. $^{28} 141-$ $\left.143{ }^{\circ} \mathrm{C}\right) ;{ }^{1} \mathrm{H} \mathrm{NMR}\left(\mathrm{CDCl}_{3}, 400 \mathrm{MHz}\right): \delta_{\mathrm{H}}=7.85(\mathrm{~s}, 1 \mathrm{H}$, triazolyl $H), 7.69(\mathrm{~d}, J 8.8 \mathrm{~Hz}, 2 \mathrm{H}, m-$ $\mathrm{Ph} H), 7.50(\mathrm{~d}, J 8.8 \mathrm{~Hz}, 2 \mathrm{H}, o-\mathrm{Ph} H), 6.81\left(\mathrm{dd}, J 18.8,11.0 \mathrm{~Hz}, 1 \mathrm{H}, \mathrm{CH}=\mathrm{CH}_{2}\right), 6.00$ (dd, $J 18.8$, $1.2 \mathrm{~Hz}, 1 \mathrm{H}$, trans $\left.\mathrm{CH}=\mathrm{CH}_{2}\right), 5.50\left(\mathrm{dd}, J 11.0,1.2 \mathrm{~Hz}, 1 \mathrm{H}\right.$, cis $\left.\mathrm{CH}=\mathrm{CH}_{2}\right) ;{ }^{13} \mathrm{C} \mathrm{NMR}\left(\mathrm{CDCl}_{3}, 100\right.$ $\mathrm{MHz}): \delta_{\mathrm{C}}=148.9(\mathrm{NCH}=C), 134.3(m-\mathrm{Ph}, 2 \mathrm{C}), 132.2(o-\mathrm{Ph}, 2 \mathrm{C}), 130.6(\mathrm{NCH}=\mathrm{C}), 129.8(\mathrm{Ph}$, $\mathrm{CN}), 126.0\left(\mathrm{CH}=\mathrm{CH}_{2}\right), 120.4(\mathrm{Ph}, \mathrm{BrC}), 116.7\left(\mathrm{CH}=\mathrm{CH}_{2}\right)$.

1-(4-Nitrophenyl)-4-vinyl-1 H-1,2,3-triazole (4k). Pale yellow solid, mp $177-179{ }^{\circ} \mathrm{C} ;{ }^{1} \mathrm{H}$ NMR $\left(\mathrm{CDCl}_{3}, 400 \mathrm{MHz}\right): \delta_{\mathrm{H}}=8.42(\mathrm{~d}, J 8.8 \mathrm{~Hz}, 2 \mathrm{H}, o-\mathrm{Ph} H), 8.00(\mathrm{~d}, J 8.8 \mathrm{~Hz}, 2 \mathrm{H}, m-\mathrm{Ph} H), 7.69(\mathrm{~s}$, $1 \mathrm{H}$, triazolyl $H), 6.80\left(\mathrm{dd}, J 18.0,10.2 \mathrm{~Hz}, 1 \mathrm{H}, \mathrm{CH}=\mathrm{CH}_{2}\right), 6.02\left(\mathrm{~d}, J 18.0 \mathrm{~Hz}, 1 \mathrm{H}\right.$, trans $\left.\mathrm{CH}=\mathrm{CH}_{2}\right)$, $5.51\left(\mathrm{~d}, J 10.2 \mathrm{~Hz}, 1 \mathrm{H}\right.$, cis $\left.\mathrm{CH}=\mathrm{CH}_{2}\right) ;{ }^{13} \mathrm{C} \mathrm{NMR}\left(\mathrm{CDCl}_{3}, 100 \mathrm{MHz}\right): \delta_{\mathrm{C}}=151.3\left(\mathrm{Ph}, \mathrm{NO}_{2} C\right), 149.1$ $(\mathrm{NCH}=C), 141.3(\mathrm{Ph}, \mathrm{CN}), 130.0(\mathrm{NCH}=\mathrm{C}), 126.4\left(\mathrm{CH}=\mathrm{CH}_{2}\right), 125.7(o-\mathrm{Ph}, 2 \mathrm{C}), 120.5(m-\mathrm{Ph}$, 2C), $117.1\left(\mathrm{CH}=\mathrm{CH}_{2}\right)$; IR (KBr): $v_{\max }=3121,1637,1606,1529,1496,1455,1350,1239,1073$, 993, 919, 775, $730 \mathrm{~cm}^{-1}$; Calcd for $\mathrm{C}_{10} \mathrm{H}_{8} \mathrm{~N}_{4} \mathrm{O}_{2}$ : C 55.55, H 3.73, N 25.91; found: C 55.23, H 3.94, N 25.76.

\section{Acknowledgements}

The authors thank the financial support from the Natural Science Foundation of China (No. 21062007), the Opening Foundation of National Research Center for Carbohydrate Synthesis (No. GJDTZX-KF-201414), the Opening Foundation of Key Laboratory of Functional Small Organic Molecule, Ministry of Education (No. KLFS-KF-201411) and the Research Program of Jiangxi Province Department of Education (No. GJJ11380).

\section{References}


1. Wamhoff, H. In Comprehensive Heterocyclic Chemistry, Katritzky, A. R.; Rees, C. W., Eds.; Pergamon: Oxford, 1984; Vol. 5.

2. Perez, J. M.; Simeone, F. J.; Saeki, Y.; Josephson, L.; Weissleder, R. J. Am. Chem. Soc. 2003, 125, 10192-10193. http://dx.doi.org/10.1021/ja036409g

3. Moses, J. E.; Moorhouse, A. D. Chem. Soc. Rev. 2007, 36, 1249-1262. http://dx.doi.org/10.1039/b613014n; PMid:17619685.

4. Rostovtsev, V. V.; Green, L. G.; Fokin, V. V.; Sharpless, K. B. Angew. Chem. Int. Ed. 2002, 41, 2596-2599. http://dx.doi.org/10.1002/1521-3773(20020715)41:14<2596::AID-ANIE2596>3.0.CO;2-4

5. Tornøe, C. W.; Christensen, C.; Meldal, M. J. Org. Chem. 2002, 67, 3057-3064. http://dx.doi.org/10.1021/jo011148j

6. Meldal, M.; Tornøe, C. W. Chem. Rev. 2008, 108, 2952-3015. http://dx.doi.org/10.1021/cr0783479

7. Kappe, C. O.; Van der Eycken, E. Chem. Soc. Rev. 2010, 39, 1280-1290. http://dx.doi.org/10.1039/b901973c

8. Hein, J. E.; Fokin, V. V. Chem. Soc. Rev. 2010, 39, 1302-1315. http://dx.doi.org/10.1039/B904091A

9. Sarmiento-Sánchez, J. I.; Ochoa-Terán, A.; Rivero, I. A. Arkivoc 2011, (ix), 177-188.

10. Testero, S. A.; Llarrull, L. I.; Fisher, J. F.; Chang, M.; Mobashery, S. Arkivoc 2011, (vii), 221-236.

11. Artyushin, O. I.; Matveeva, E. V.; Bushmarinov, I. S.; Odinets, I. L. Arkivoc 2012, (iv) 252263.

12. López-Ruiz, H.; de la Cerda-Pedro, J. E.; Rojas-Lima, S.; Pérez-Pérez, I.; RodríguezSónchez, B. V.; Santillan, R.; Coreñoc, O. Arkivoc 2013, (iii), 139-164.

13. Verma, M.; Luxami, V.; Paul, K. Arkivoc 2015, (vii), 28-41. http://dx.doi.org/10.3998/ark.5550190.p009.175

14. Wouters, G.; Smets, G. Makromol. Chem. 1982, 183, 1861-1868. http://dx.doi.org/10.1002/macp.1982.021830805

15. Feldman, A.K.; Colasson, B.; Fokin, V. V. Org. Lett. 2004, 6, 3897-3899. http://dx.doi.org/10.1021/o1048859z

16. Thibault, R. J.; Takizawa, K.; Lowenhielm, P.; Helms, B.; Mynar, J. L.; Fréchet, J. M. J.; Hawker, C. J. J. Am. Chem. Soc. 2006, 128, 12084-12085. http://dx.doi.org/10.1021/ja0648209

17. Takizawa, K.; Nulwala, H.; Thibault, R. J.; Lowenhielm, P.; Yoshinaga, K.; Wooley, K. L.; Hawker, C. J. J. Polym. Sci. Part A: Polym. Chem. 2008, 46, 2897-2812. http://dx.doi.org/10.1002/pola.22627

18. Nulwala, H.; Burke, D. J.; Khan, A.; Serrano, A.; Hawker, C. J. Macromolecules 2010, 43, 5474-5477. 
http://dx.doi.org/10.1021/ma100011x

19. He, A. Y.; Sheng, S. R.; Huang, Z. Z.; Li, L.; Cai, M. Z. J. Heterocycl. Chem. 2014, 51, 1862-1865.

http://dx.doi.org/10.1002/jhet.1857

20. Brahma, K.; Achari, B.; Chinmay Chowdhury, C. Synthesis 2013, 45, 545-555. http://dx.doi.org/10.1055/s-0032-1316839

21. Reddy, M. N.; Swamy, K. C. K. Eur. J. Org. Chem. 2012, 2013-2022. http://dx.doi.org/10.1002/ejoc.201101816

22. Carcenac, Y.; David-Quillot, F.; Abarbri, M.; Duchêne, A.; Thibonnet, J. Synthesis 2013, 45, 633-648.

http://dx.doi.org/10.1055/s-0032-1318112

23. Dickerson, T. J.; Reed, N. N.; Janda, K. D. Chem. Rev. 2002, 102, 3325-3344.

http://dx.doi.org/10.1021/cr010335e

24. Toy, P. H.; Janda, K. D. Acc. Chem. Res. 2000, 33, 546-554.

http://dx.doi.org/10.1021/ar990140h

25. Zhang, X. L.; Sheng, S. R.; Liu, X.-L. Liu, X. L. Arkivoc 2007, (xiii), 79-86.

26. Liu, X. L.; Wang, Q. Y.; Sheng, S. R.; Xu, C.; Cai, M. Z. Synth. Commun. 2008, 38, 33383345 .

http://dx.doi.org/10.1080/00397910802136672

27. Shin, J. A.; Lim, Y. G.; Lee, K. H. J. Org. Chem. 2012, 77, 4117-4122.

http://dx.doi.org/ 10.1021/j03000095

28. Cardoen, H.; Toppet, S.; Smets, G.; L’abbé, G. J. Heterocycl. Chem. 1972, 9, 971-972.

http://dx.doi.org/10.1002/jhet.5570090444 